\title{
Kombinatorische Festphasensynthese von multivalenten cyclischen Neoglycopeptiden**
}

\author{
Valentin Wittmann* und Sonja Seeberger \\ Professor Horst Kessler gewidmet
}

Die molekulare Erkennung von Kohlenhydraten durch Kohlenhydrat-bindende Proteine (Lectine) ist die Basis zahlreicher interzellulärer Erkennungsprozesse. ${ }^{[1]}$ Hochaffine Lectinliganden sind von großem medizinischem Interesse in der Diagnostik und Manipulation solcher Prozesse. ${ }^{[2]}$ Individuelle Kohlenhydratepitope (in der Regel Mono- bis Pentasaccharide) werden von Lectinen allerdings meist nur mit niedriger Affinität (Dissoziationskonstanten im milli- bis mikromolaren Bereich) und zum Teil geringer Spezifität gebunden. ${ }^{[3]}$ Da viele membranständige Lectine mehrere

[*] Dr. V. Wittmann, Dipl.-Chem. S. Seeberger Institut für Organische Chemie

Johann Wolfgang Goethe-Universität

Marie-Curie-Straße 11, 60439 Frankfurt (Deutschland)

Fax: $(+49)$ 69-798-29148

E-mail:wittmann@chemie.uni-frankfurt.de

[**] Diese Arbeit wurde von der Deutschen Forschungsgemeinschaft gefördert. Wir danken Prof. Joachim W. Engels für seine Unterstützung und der Degussa-Hüls AG für Aminosäurespenden. Verwendete Abkürzungen werden in Lit. [25] erwähnt.

$\square$ Hintergrundinformationen zu diesem Beitrag sind im WWW unter http://www.angewandte.de zu finden oder können vom Autor angefordert werden.

Konstanzer Online-Publikations-System (KOPS)

URL: http://www.ub.uni-konstanz.de/kops/volltexte/2007/4377/

URN: http://nbn-resolving.de/urn:nbn:de:bsz:352-opus-43775 
Bindungsstellen aufweisen oder in oligomerer oder gehäufter Form vorliegen, ${ }^{[4]}$ ist die Erzeugung multivalenter Kohlenhydratderivate ein Erfolg versprechender Ansatz, um zu effektiven Lectinliganden zu gelangen. ${ }^{[5,6]}$

Verschiedene Strategien wurden beschrieben, um die für eine hohe Avidität eines multivalenten Liganden notwendige Bildung einer ausreichenden Zahl von Wechselwirkungen zu erreichen. Glycopolymere ${ }^{[7]}$ können beispielsweise große Bereiche von Zelloberflächen abdecken und mehrere membranständige Lectine überbrücken („statistische“ Multivalenz). Kleine oligovalente Kohlenhydratderivate (Minicluster) ${ }^{[6]}$ dagegen binden bevorzugt an mehrere räumlich benachbarte Bindungsstellen eines einzelnen (oligomeren) Lectins und können an Lectine mit bekannter 3DStruktur angepasst werden (,gezielte“ Multivalenz). ${ }^{[8]}$ Sind die Kohlenhydratepitope dabei durch ein starres Gerüst miteinander verbunden, erhält man besonders af-
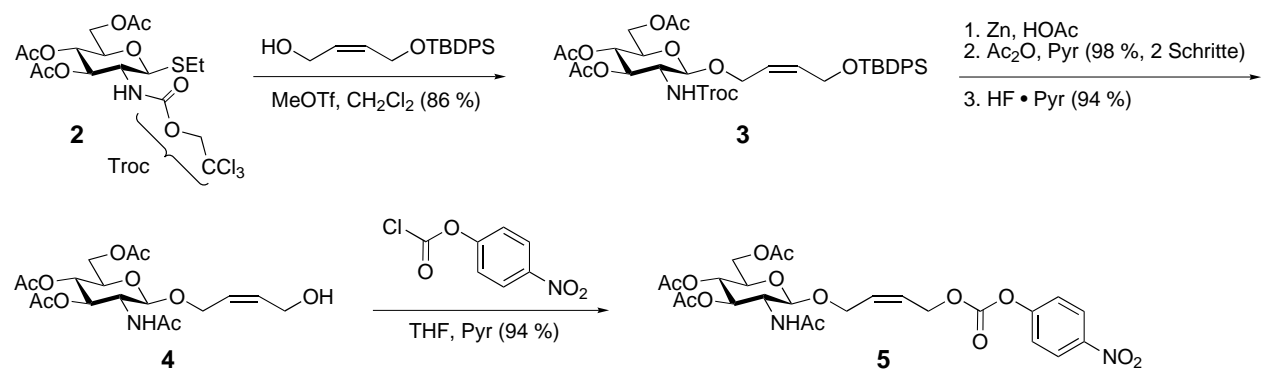

Schema 1. Synthese des als $p$-Nitrophenylcarbonat aktivierten Kohlenhydratderivats 5. seitenkettencyclisierten Peptiden Palladium-katalysiert wieder abspalten. Dadurch reduziert sich die beim Arbeiten nach der Split-Mix-Methode notwendige Analyse der an einzelne Harzkugeln gebundenen Verbindungen auf eine automatisierte Mikrosequenzierung (Edman-Abbau) unter Standardbedingungen.

Schema 1 zeigt die Synthese des als $p$-Nitrophenylcarbonat aktivierten Kohlenhydrat-Linker-Derivats 5, das zur Ankupplung von $N$-Acetylglucosaminresten an die Cyclopeptide diente. Umsetzung des Glycosyldonors 2 $2^{[15]}$ mit mono- fine Liganden - vorausgesetzt, die

für eine mehrzähnige Bindung notwendige Geometrie liegt vor. $^{[5]}$ Zudem können konformativ eingeschränkte Minicluster prinzipiell zwischen verschiedenen multivalenten Lectinen mit gleicher Kohlenhydratspezifität, aber unterschiedlicher Anordnung der Bindungsstellen differenzieren. Ist die räumliche Struktur des zu untersuchenden Lectins unbekannt, muss - insbesondere beim Einsatz konformativ eingeschränkter „Linker“ - eine große Zahl an potentiellen Liganden synthetisiert und getestet werden, um die erforderliche Anordnung der Zuckerreste zu ermitteln.

Wir beschreiben hier ein Konzept zur Generierung von Bibliotheken aus konformativ eingeschränkten Miniclustern, das folgende Schritte umfasst: a) „Split-Mix“-Synthese ${ }^{[9]}$ einer Bibliothek aus Gerüstmolekülen mit SeitenkettenAminogruppen in unterschiedlicher Zahl und räumlicher Anordnung, b) Ankupplung mehrerer identischer Kohlenhydratliganden an die Aminogruppen.

Als Gerüste zur multivalenten Präsentation der Kohlenhydratliganden haben wir cyclische Peptide der allgemeinen Formel 1 (Bal $=\beta$-Alanin) gewählt, in denen die Reste Xaa kombinatorisch variiert werden. ${ }^{[10]}$ Durch Einsatz kommerziell erhältlicher D- und L-konfigurierter Aminosäuren lässt

Boc-Lys-Xaa-Xaa-Xaa-Xaa-Xaa-Xaa-Glu-Bal-NH-

1

sich leicht ein hohes $\mathrm{Ma}$ an konformativer Diversität erzeugen. ${ }^{[1]}$ Diaminosäuren mit Ddv-geschützen ${ }^{[12]}$ Seitenketten variabler Länge dienen zur Anbindung der Zucker. Hierzu wurde ein neuer, auf der Aloc-Schutzgruppe ${ }^{[13]}$ basierender Linker vom Urethantyp entwickelt. Im Unterschied zu Glycosylierungen mit festphasengebundenen Peptiden $^{[14]}$ verläuft die Urethanbildung in praktisch quantitativer Ausbeute. Außerdem lassen sich im Anschluss an einen Bindungstest an fester Phase die Kohlenhydrate von den
TBDPS-geschütztem cis-But-2-en-1,4-diol unter Methyltriflat-Aktivierung lieferte das $\beta$-konfigurierte $\mathrm{O}$-Glycosid $\mathbf{3}$ in hoher Ausbeute. Austausch der Troc-Schutzgruppe gegen eine Acetylgruppe und anschließende Abspaltung der TBDPS-Gruppe mit HF-Pyridin-Komplex führten zum $N$ Acetylglucosaminderivat 4. Kondensation mit Chlorameisensäure- $p$-nitrophenylester ergab schließlich das aktivierte Carbonat 5 als eine kristalline, bei Raumtemperatur lagerbare Substanz (Tabelle 1).

\section{Tabelle 1. Ausgewählte physikalische Daten von $\mathbf{5}$ und 12.}

5: $R_{\mathrm{f}}=0.43$ (Kieselgel, EtOAc); Schmp. $133^{\circ} \mathrm{C}($ EtOAc $/ n-H e x a n) ;{ }^{1} \mathrm{H}-$ NMR ( $\left.400 \mathrm{MHz}, \mathrm{CDCl}_{3}, 300 \mathrm{~K}, \mathrm{TMS}\right): \delta=8.29-8.25(\mathrm{~m}, 2 \mathrm{H}$, aromat. $\mathrm{H})$, $7.40-7.36(\mathrm{~m}, 2 \mathrm{H}$, aromat. H), 5.85-5.75 (m, 2H, Vinyl-H), $5.64(\mathrm{~d}, J=$ $8.7 \mathrm{~Hz}, 1 \mathrm{H}, \mathrm{NH}), 5.28(\mathrm{dd}, J=9.3,10.6 \mathrm{~Hz}, 1 \mathrm{H}, \mathrm{H}-3), 5.05(\mathrm{dd}, J=9.3$, $10.0 \mathrm{~Hz}, 1 \mathrm{H}, \mathrm{H}-4), 4.90-4.79$ (m, $2 \mathrm{H}$, Allyl-H), $4.75(\mathrm{~d}, J=8.3 \mathrm{~Hz}, 1 \mathrm{H}$, H-1), $4.44-4.29$ (m, 2H, Allyl-H), 4.22 (dd, $J=4.7,12.3 \mathrm{~Hz}, 1 \mathrm{H}, \mathrm{H}-6 \mathrm{a})$, $4.13(\mathrm{dd}, J=2.5,12.3 \mathrm{~Hz}, 1 \mathrm{H}, \mathrm{H}-6 \mathrm{~b}), 4.83$ (ddd, $J=8.3,8.7,10.6 \mathrm{~Hz}, 1 \mathrm{H}$, $\mathrm{H}-2$ ), 3.69 (ddd, $J=2.5,4.7,10.0 \mathrm{~Hz}, 1 \mathrm{H}, \mathrm{H}-5), 2.06\left(\mathrm{~s}, 3 \mathrm{H}, \mathrm{C}(\mathrm{O}) \mathrm{CH}_{3}\right), 2.01$ $\left(\mathrm{s}, 3 \mathrm{H}, \mathrm{C}(\mathrm{O}) \mathrm{CH}_{3}\right), 2.00\left(\mathrm{~s}, 3 \mathrm{H}, \mathrm{C}(\mathrm{O}) \mathrm{CH}_{3}\right), 1.93\left(\mathrm{~s}, 3 \mathrm{H}, \mathrm{C}(\mathrm{O}) \mathrm{CH}_{3}\right) ;{ }^{13} \mathrm{C}-$ NMR $\left(100 \mathrm{MHz}, \mathrm{CDCl}_{3}, 300 \mathrm{~K}, \mathrm{TMS}\right): \delta=170.8,170.6,170.2,169.3,155.4$, $152.4,145.4,131.0$ u. 125.8 (olefin. C), 125.3 u. 121.8 (aromat. CH), 99.5 (C1), 72.2 (C-3), 71.8 (C-5), 68.6 (C-4), 64.6 u. $64.2\left(\mathrm{CH}_{2}{ }^{\text {allyl }}\right), 62.0$ (C-6), 54.7 (C-2), $23.3\left(\mathrm{CH}_{3}\right), 20.67\left(\mathrm{CH}_{3}\right), 20.61\left(\mathrm{CH}_{3}\right), 20.55\left(\mathrm{CH}_{3}\right) ; \mathrm{C}, \mathrm{H}, \mathrm{N}$-Analyse: ber. für $\mathrm{C}_{25} \mathrm{H}_{30} \mathrm{~N}_{2} \mathrm{O}_{14}$ : C 51.55, H 5.19, N 4.81; gef. C 51.52, H 5.28, N 4.79 12: ${ }^{1} \mathrm{H}-\mathrm{NMR}\left(600 \mathrm{MHz}, \mathrm{H}_{2} \mathrm{O} / \mathrm{D}_{2} \mathrm{O} 9: 1,298 \mathrm{~K}\right)$ : Boc-Lys ${ }^{1}: \delta=6.767(\alpha \mathrm{NH})$, $3.917(\alpha \mathrm{H}), 1.565\left(\beta \mathrm{H}_{2}\right), 1.27-1.17\left(\gamma \mathrm{H}_{2}\right), 1.379\left(\delta \mathrm{H}_{2}\right), 3.083$ u. $3.003\left(\varepsilon \mathrm{H}_{2}\right)$, $7.688(\varepsilon \mathrm{NH}), 1.304(\mathrm{Boc}) ;$ Orn: $\delta=8.262(\alpha \mathrm{NH}), 4.254(\alpha \mathrm{H}), 1.722$ u. 1.633 $\left(\beta \mathrm{H}_{2}\right), 1.47-1.38\left(\gamma \mathrm{H}_{2}\right), 3.023\left(\delta \mathrm{H}_{2}\right), 6.791(\delta \mathrm{NH}) ; \mathrm{Gly}^{3}: \delta=8.201(\mathrm{NH})$, 3.855 u. $3.788\left(\alpha \mathrm{H}_{2}\right) ; \mathrm{Ala}^{4}: \delta=7.999(\mathrm{NH}), 4.203(\alpha \mathrm{H}), 1.277\left(\beta \mathrm{H}_{3}\right) ; \mathrm{D}-\mathrm{Lys}^{5}$ : $\delta=8.148(\alpha \mathrm{NH}), 4.185(\alpha \mathrm{H}), 1.690$ u. $1.615\left(\beta \mathrm{H}_{2}\right), 1.248$ u. $1.215\left(\gamma \mathrm{H}_{2}\right)$, $1.382\left(\delta \mathrm{H}_{2}\right), 2.998\left(\varepsilon \mathrm{H}_{2}\right), 6.733(\varepsilon \mathrm{NH})$; Orn: $\delta=8.316(\alpha \mathrm{NH}), 4.254(\alpha \mathrm{H})$, 1.731 u. $1.633\left(\beta \mathrm{H}_{2}\right), 1.478$ u. $1.420\left(\gamma \mathrm{H}_{2}\right), 3.032\left(\delta \mathrm{H}_{2}\right), 6.767(\delta \mathrm{NH}) ; \mathrm{D}^{-V a l^{7}}$ : $\delta=8.136(\mathrm{NH}), 3.982(\alpha \mathrm{H}), 2.041(\beta \mathrm{H}), 0.820$ u. $0.786\left(2 \gamma \mathrm{H}_{3}\right)$; $\mathrm{Glu}^{8}: \delta=$ $8.201(\mathrm{NH}), 4.107(\alpha \mathrm{H}), 1.831$ u. $1.999\left(\beta \mathrm{H}_{2}\right), 2.201$ u. $2.136\left(\gamma \mathrm{H}_{2}\right) ; \mathrm{Bal}^{9}: \delta=$ $8.049(\mathrm{NH}), 2.41-2.33\left(\alpha \mathrm{H}_{2}\right), 3.40-3.30\left(\beta \mathrm{H}_{2}\right) ; 3$ äquival. GlcNAc: $\delta=$ $8.103(\mathrm{NH}), 4.427$ (H-1), 3.588 (H-2), 3.429 (H-3), 3.38-3.31 (H-4, H-5), 3.810 u. 3.651 (2 H-6), 1.927 (Ac); $5.72-5.60$ (6 Vinyl-H), 4.52-4.48 u. 4.30-4.18 (je 6 Allyl-H); ESI-MS $\left(M+\mathrm{H}^{+}\right)$: ber. 1963.0, gef. 1963.7 
Der Aufbau der cyclischen Neoglycopeptide wurde am Beispiel der Modellverbindung 12 optimiert (Schemata 2 und 3, Tabelle 1). Die Anknüpfung der ersten Aminosäure an das Trägermaterial (TentaGel) erfolgte mit dem Sieber-Linker. ${ }^{[16]}$ Dadurch war es möglich, auf jeder Stufe der Reaktionssequenz das jeweilige Produkt durch Behandlung mit einer 1-proz. Lösung von TFA in Dichlormethan abzuspalten $(\rightarrow \mathbf{7}-\mathbf{9}, \mathbf{1 1}, 12)$ und den Syntheseverlauf durch analytische HPLC (Abbildung 1) und anschließende ESI-MS zu verfolgen. Die Synthese des linearen Peptids erfolgte nach der Fmoc-Strategie; ${ }^{[17]}$ lediglich im letzten Kupplungsschritt kam eine Boc-geschützte Aminosäure zum Einsatz. Zum Schutz der $\omega-\mathrm{NH}_{2}$-Gruppen von Lysin und Ornithin bevorzugten wir die Ddv-Gruppe, ${ }^{[12]}$ die sich von der häufig eingesetzten DdeGruppe $^{[18]}$ durch eine höhere Stabilität unter den Bedingungen der Fmoc-Abspaltung auszeichnet. Zur Vorbereitung des Cyclisierungsschritts wurden der Allylester und die Aloc-

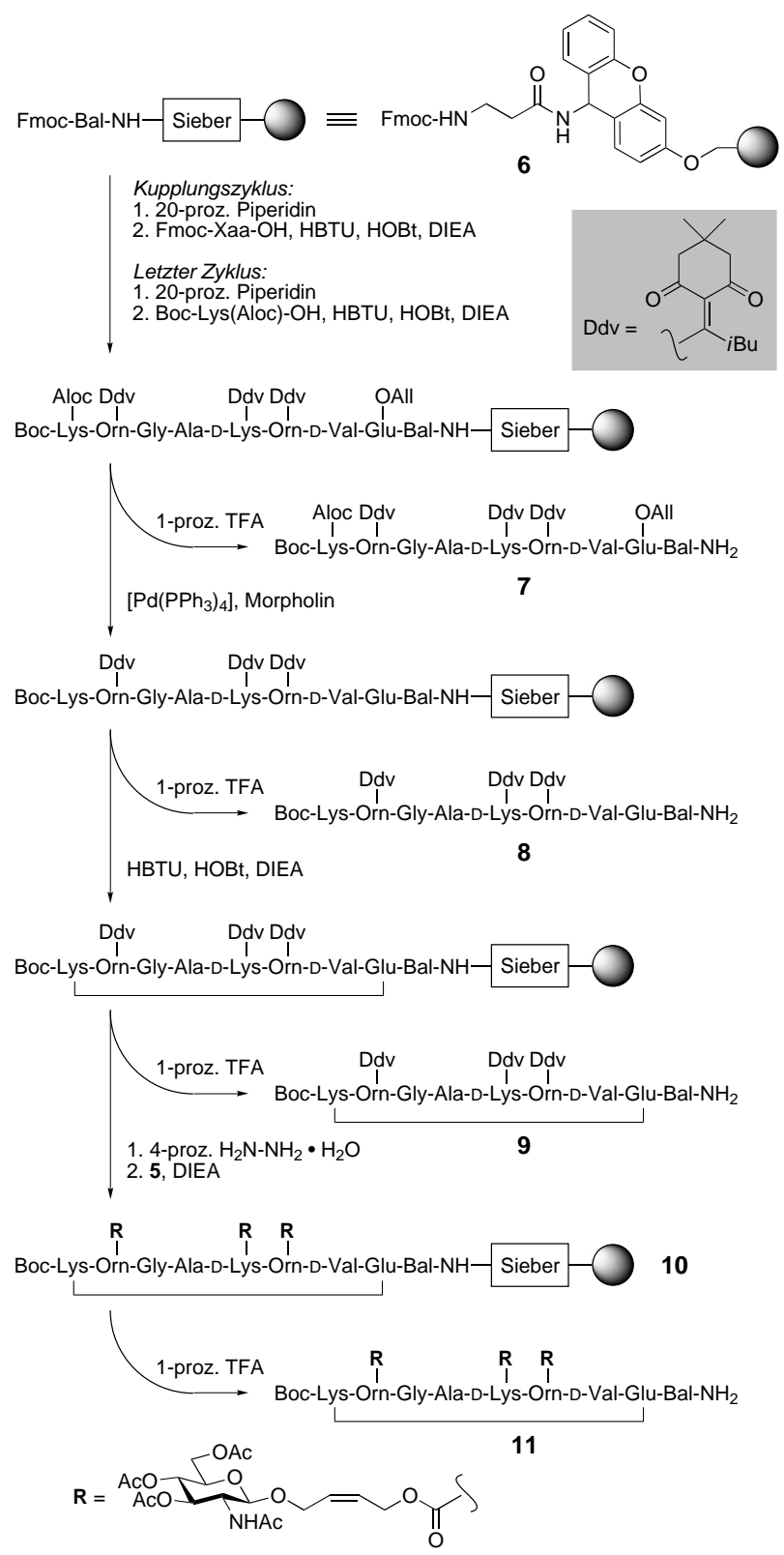

Schema 2. Festphasensynthese des cyclischen Neoglycopeptids 11.
A)

10

B)

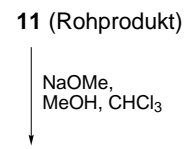

R-HN 1. $\mathrm{NaOMe}, \mathrm{MeOH}$
2. 1 -proz. TFA

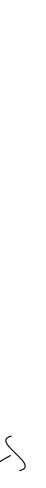

12

Schema 3. Synthese des entschützten Neoglycopeptids 12.

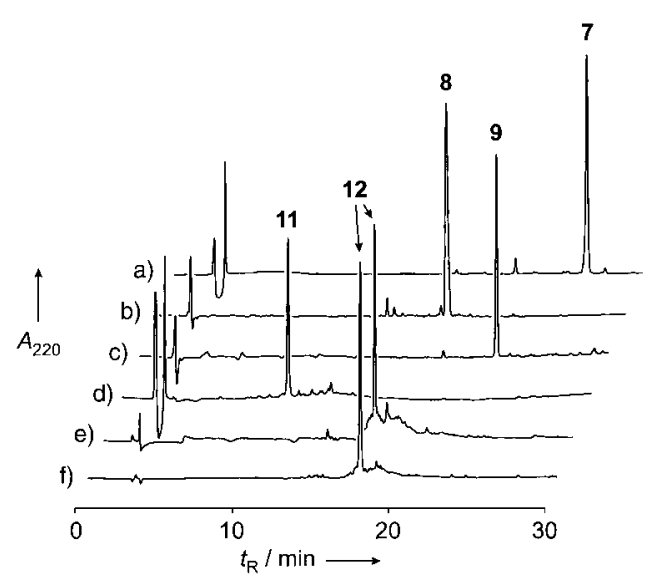

Abbildung 1. RP-HPLC-Analyse der rohen Peptide 7-9, 11 und 12, erhalten nach Schema 2 (a-d), Schema 3, Weg A (e) und Schema 3, Weg B (f).

Schutzgruppe simultan durch Palladium(0)-katalysierte Allylübertragung auf Morpholin als Abfangnucleophil entfernt. ${ }^{[13]}$

Zugabe einer Mischung aus HBTU, HOBt und DIEA führte zum gewünschten Cyclopeptid, das nach Abspaltung vom Harz in ausgezeichneter Reinheit vorlag (Abbildung 1c). Nebenprodukte, hervorgerufen durch die Reaktion von Kupplungsreagens mit der freien Aminogruppe ${ }^{[19]}$ (Guanylierung), wurden nicht beobachtet. Andere Cyclisierungsmethoden ( $N, N^{\prime}$-Diisopropylcarbodiimid/HOBt oder Aktivierung der Carbonsäure als Pfp-Ester ${ }^{[20]}$ ) waren deutlich weniger effektiv. Anschließend wurden die Ddv-Gruppen abgespalten, wobei sich in dem von uns angewandten Batch-Verfahren die Verwendung von 4-proz. Hydrazinhydrat als vorteilhafter gegenüber der in der Literatur empfohlenen ${ }^{[12]} 2$-proz. Lösung herausstellte. ${ }^{[21]}$

Die Ankupplung der Zucker erfolgte durch Zusatz eines Überschusses (5 Äquiv. pro freie $\mathrm{NH}_{2}$-Gruppe) an $\mathbf{5}$ in Gegenwart von DIEA. Nach beendeter Reaktion wurde die Abwesenheit freier Aminogruppen durch den Kaiser-Test ${ }^{[22]}$ und Zugabe von Bromphenolblau ${ }^{[23]}$ verifiziert. Zur Desacetylierung der Zucker wurde $\mathbf{1 0}$ mit einer Lösung von Natriummethanolat in Methanol behandelt (Schema 3, 
Weg A). Nach Abspaltung vom Harz erhielt man das in Abbildung 1e gezeigte HPL-Chromatogramm, in dem neben dem gewünschten Glycopeptid 12 deutliche Mengen an Nebenprodukten erkennbar sind. Da deren Auftreten möglicherweise mit der Säurelabilität von 12 verknüpft ist ${ }^{[24]}$ und somit nicht die tatsächlichen Verhältnisse am Harz widergespiegelt werden, haben wir zum Vergleich die Desacetylierung nach der Abspaltung vom Träger vorgenommen (Schema 3, Weg B). Das Chromatogramm des so erhaltenen Rohproduktes (Abbildung $1 \mathrm{f}$ ) zeigt nur noch geringe Mengen an Nebenprodukt und demonstriert die hohe Effizienz der gesamten Synthese.

Um $\mathrm{zu}$ verifizieren, dass die beschriebenen Reaktionsbedingungen, speziell die des kritischen Cyclisierungsschritts,

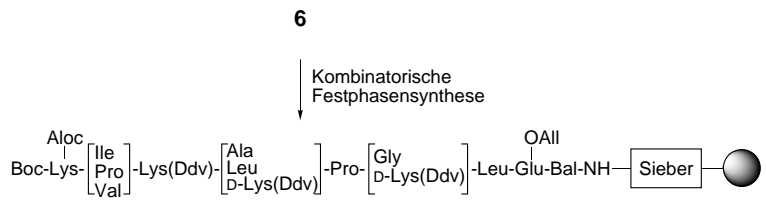

13

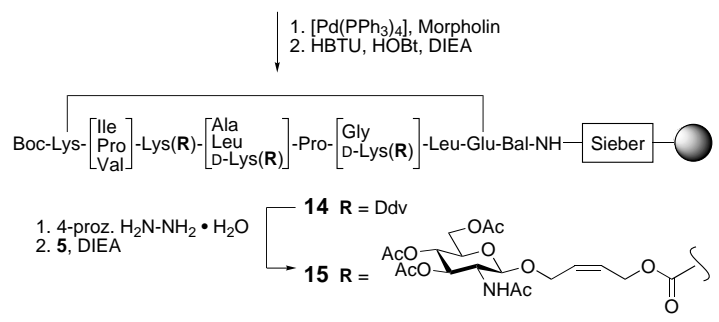

Schema 4. Split-Mix-Synthese der Neoglycopeptid-Bibliothek 15. An den drei mit eckigen Klammern gekennzeichneten Positionen wurde das Syntheseharz auf zwei oder drei Reaktionsgefäße verteilt und jeweils eine der angegebenen Aminosäuren angekuppelt. auch auf andere Peptidsequenzen übertragbar sind, synthetisierten wir die aus 18 Verbindungen bestehende Neoglycopeptid-Bibliothek 15 nach der Split-Mix-Methode (Schema 4). Wiederum wurde der Verlauf der Synthese durch Entnahme kleiner Harzproben und Analyse der Abspaltprodukte durch HPLC in Kombination mit ESI-MS verfolgt (Abbildung 2, Tabelle 2). In allen Chromatogrammen konn-
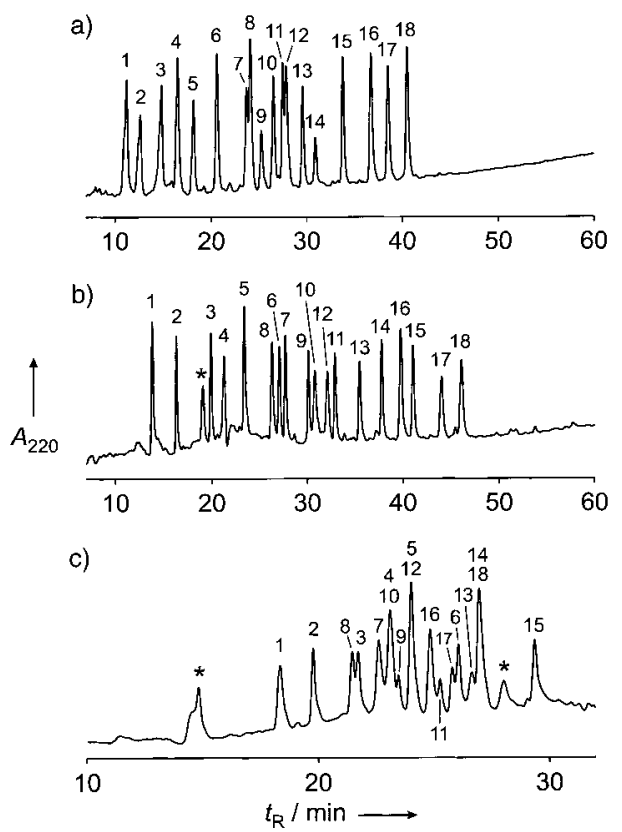

Abbildung 2. RP-HPLC-Analyse der nach Behandlung von a) 13, b) 14 und c) 15 mit 1-proz. TFA erhaltenen Verbindungsbibliotheken. Die Zuordnung der Peaks erfolgte massenspektrometrisch und ist in Tabelle 2 angegeben. Peaks, hervorgerufen von Peptidderivaten gleicher Sequenz, tragen identische Nummern. Die mit Sternchen gekennzeichneten stammen von nichtpeptidischen Verunreinigungen.

Tabelle 2. Berechnete und experimentell gefundene Massen der nach Behandlung von 15 mit 1-proz. TFA erhaltenen Bibliothek von cyclischen Neoglycopeptiden (die Peaknummern entsprechen der Zuordnung in Abbildung 2c).

\begin{tabular}{|c|c|c|c|}
\hline \multirow[t]{2}{*}{ Peak } & \multirow[t]{2}{*}{ Verbindung ${ }^{[\mathrm{a}]}$} & \multicolumn{2}{|c|}{$[M+\mathrm{H}]^{+}$} \\
\hline & & ber. & gef. \\
\hline 1 & cyclo[Boc-Lys-Pro-Lys( $(\mathbf{R})$-Ala-Pro-Gly-Leu-Glu]-Bal-NH ${ }_{2}$ & 1434.7 & 1435.4 \\
\hline 2 & cyclo[Boc-Lys-Val-Lys $(\mathbf{R})$-Ala-Pro-Gly-Leu-Glu]-Bal-NH ${ }_{2}$ & 1436.7 & 1437.3 \\
\hline 8 & cyclo[Boc-Lys-Pro-Lys( $\mathbf{R})$-D-Lys( $(\mathbf{R})$-Pro-Gly-Leu-Glu]-Bal-NH ${ }_{2}$ & 1934.9 & 1935.7 \\
\hline 3 & cyclo[Boc-Lys-Ile-Lys( $(\mathbf{R})$-Ala-Pro-Gly-Leu-Glu]-Bal-NH ${ }_{2}$ & 1450.8 & 1451.2 \\
\hline 7 & cyclo[Boc-Lys-Pro-Lys( $(\mathbf{R})$-Ala-Pro-D-Lys $(\mathbf{R})$-Leu-Glu]-Bal-NH ${ }_{2}$ & 1949.0 & 1950.0 \\
\hline 4 & cyclo[Boc-Lys-Pro-Lys $(\mathbf{R})-L e u-P r o-G l y-L e u-G l u]-B a l-\mathrm{NH}_{2}$ & 1476.8 & 1477.4 \\
\hline 10 & cyclo[Boc-Lys-Val-Lys(R)-D-Lys( $(\mathbf{R})$-Pro-Gly-Leu-Glu]-Bal-NH ${ }_{2}$ & 1937.0 & 1938.0 \\
\hline 9 & $\begin{array}{l}\text { cyclo }\left[\text { Boc-Lys-Val-Lys }(\mathbf{R}) \text {-Ala-Pro-D-Lys }(\mathbf{R}) \text {-Leu-Glu]-Bal-NH } \mathrm{N}_{2} \text { oder }\right. \\
\text { cyclo[Boc-Lys-Ile-Lys }(\mathbf{R}) \text {-D-Lys }(\mathbf{R}) \text {-Pro-Gly-Leu-Glu]-Bal-NH }{ }_{2}\end{array}$ & 1951.0 & 1952.0 \\
\hline 5 & cyclo[Boc-Lys-Val-Lys( $\mathbf{R})$-Leu-Pro-Gly-Leu-Glu]-Bal-NH ${ }_{2}$ & 1478.8 & 1479.4 \\
\hline 12 & $\begin{array}{l}\text { cyclo[Boc-Lys-Ile-Lys }(\mathbf{R}) \text {-D-Lys }(\mathbf{R})-\text { Pro-Gly-Leu-Glu]-Bal-NH } \mathrm{N}_{2} \text { oder } \\
\text { cyclo[Boc-Lys-Val-Lys }(\mathbf{R}) \text {-Ala-Pro-D-Lys }(\mathbf{R})-L e u-G l u]-B a l-\mathrm{NH}_{2}\end{array}$ & 1951.0 & 1952.0 \\
\hline 16 & cyclo $\left[\right.$ Boc-Lys-Pro-Lys $(\mathbf{R})$-D-Lys( $(\mathbf{R})$-Pro-D-Lys $(\mathbf{R})$-Leu-Glu]-Bal- $\mathrm{NH}_{2}$ & 2449.2 & 2450.1 \\
\hline 11 & cyclo[Boc-Lys-Ile-Lys $(\mathbf{R})$-Ala-Pro-D-Lys $(\mathbf{R})$-Leu-Glu]-Bal-NH ${ }_{2}$ & 1965.0 & 1966.2 \\
\hline 13 & cyclo[Boc-Lys-Pro-Lys( $(\mathbf{R})$-Leu-Pro-D-Lys( $(\mathbf{R})$-Leu-Glu]-Bal-NH ${ }_{2}$ & 1991.0 & 1991.8 \\
\hline 14 & cyclo[Boc-Lys-Val-Lys(R)-Leu-Pro-D-Lys(R)-Leu-Glu]-Bal-NH ${ }_{2}$ & 1993.0 & 1993.6 \\
\hline 18 & cyclo[Boc-Lys-Ile-Lys(R)-D-Lys(R)-Pro-D-Lys(R)-Leu-Glu]-Bal-NH ${ }_{2}$ & 2465.2 & 2465.9 \\
\hline 15 & cyclo[Boc-Lys-Ile-Lys $(\mathbf{R})$-Leu-Pro-D-Lys $(\mathbf{R})$-Leu-Glu]-Bal-NH ${ }_{2}$ & 2007.0 & 2008.0 \\
\hline
\end{tabular}

[a] $\mathbf{R}={ }_{\mathrm{NHAC}}^{\mathrm{AcO}}=\prod_{\mathrm{AcO}}^{\mathrm{OAc}}$ 
ten die erwarteten 18 Produkte identifiziert werden. Es zeigte sich, dass alle Peptide ohne nennenswerte Nebenproduktbildung cyclisierten. Insbesondere konnten wir bei der Analyse von 14 massenspektrometrisch weder Edukte noch lineare oder cyclische Dimere detektieren.

Die vorgestellte Synthesestrategie eignet sich zum effizienten Aufbau von Bibliotheken aus Neoglycopeptiden, die beliebige Kohlenhydratliganden in unterschiedlicher Anzahl und unterschiedlichen Distanzen zueinander präsentieren. Die konvergente Vorgehensweise erlaubt es, ohne großen Aufwand mit einer einmal bereitgestellten Cyclopeptidbibliothek durch Ankuppeln entsprechender Kohlenhydratliganden verschiedene Lectine $\mathrm{zu}$ untersuchen.

\section{Experimentelles}

Die Festphasenpeptidsynthese erfolgte an NovaSyn-TG-Sieber-Harz (Novabiochem) (Abkürzung: Sieber-TG) nach üblichen Vorschriften ${ }^{[17]}(\mathrm{Be}-$ ladung nach Immobilisierung der ersten Aminosäure: $0.17 \mathrm{mmol} \mathrm{g}^{-1}$ ). Kupplungen wurden in NMP durchgeführt. Zur Abspaltung wurden Festphasenaliquote (ca. $5 \mathrm{mg}$ ) wiederholt mit TFA $/ i \mathrm{Pr}_{3} \mathrm{SiH} / \mathrm{CH}_{2} \mathrm{Cl}_{2}$ (1:1:98) behandelt. Produkte, die in der Abspaltlösung schwerlöslich waren, wurden mit einem Lösungsmittel von der festen Phase gewaschen. Vor dem Einengen wurden die Abspaltlösungen mit Pyridin neutralisiert. Analytische HPLC erfolgte an C18-RP-Säulen $(250 \times 4 \mathrm{~mm})$ mit linearen Gradienten von Acetonitril in Wasser/0.1-proz. TFA bei einer Fließgeschwindigkeit von $1 \mathrm{mLmin}^{-1}$. Produktpeaks wurden durch ESI-MS charakterisiert. Experimentelle Details zur Synthese von 8, 9, 11 und 12 sind den Hintergrundinformationen zu entnehmen.

Eingegangen am 16. Mai, veränderte Fassung am 4. August 2000 [Z15129]

[1] Essentials of Glycobiology (Hrsg.: A. Varki, R. Cummings, J. Esko, H. Freeze, G. Hart, J. Marth), Cold Spring Harbor Lab Press, Cold Spring Harbor, 1999.

[2] a) Y. C. Lee, R. T. Lee, J. Biomed. Sci. 1996, 3, 221-237; b) J. M. Gardiner, Expert Opin. Invest. Drugs 1998, 7, 405-411; c) C. A. Lingwood, Curr. Opin. Chem. Biol. 1998, 2, 695-700.

[3] a) H.-J. Gabius, Eur. J. Biochem. 1997, 243, 543-576; b) H. Lis, N. Sharon, Chem. Rev. 1998, 98, 637-674.

[4] J. M. Rini, Annu. Rev. Biophys. Biomol. Struct. 1995, 24, 551-577.

[5] M. Mammen, S.-K. Choi, G. M. Whitesides, Angew. Chem. 1998, 110, 2908-2953; Angew. Chem. Int. Ed. 1998, 37, 2754-2794.

[6] a) R. Roy, Curr. Opin. Struct. Biol. 1996, 6, 692-702; b) L. L. Kiessling, N. L. Pohl, Chem. Biol. 1996, 3, 71-77; c) Neoglycoconjugates. Preparation and Applications (Hrsg.: Y. C. Lee, R. T. Lee), Academic Press, San Diego, 1994.

[7] a) G. B. Sigal, M. Mammen, G. Dahmann, G. M. Whitesides, J. Am. Chem. Soc. 1996, 118, 3789-3800; b) R. Roy, Trends Glycosci. Glycotechnol. 1996, 8, 79-99; c) M. Kanai, K. H. Mortell, L. L. Kiessling, J. Am. Chem. Soc. 1997, 119, $9931-9932$.

[8] a) P. I. Kitov, J. M. Sadowska, G. Mulvery, G. D. Armstrong, H. Ling, N. S. Pannu, R. J. Read, D. R. Bundle, Nature 2000, 403, 669-672; b) E. Fan, Z. Zhang, W. E. Minke, Z. Hou, C. L. M. J. Verlinde, W. G. J. Hol, J. Am. Chem. Soc. 2000, 122, 2663-2664.

[9] K. S. Lam, M. Lebl, V. Krchňák, Chem. Rev. 1997, 97, 411-448.

[10] Zur Verwendung cyclischer Peptide als Gerüste siehe: a) U. Sprengard, M. Schudok, W. Schmidt, G. Kretzschmar, H. Kunz, Angew. Chem. 1996, 108, 359-362; Angew. Chem. Int. Ed. Engl. 1996, 35, $321-324$; b) H. Franzyk, M. K. Christensen, R. M. Jørgensen, M. Meldal, H. Cordes, S. Mouritsen, K. Bock, Bioorg. Med. Chem. 1997, 5 , $21-40$; c) G. Tuchscherer, M. Mutter, Pure Appl. Chem. 1996, 68, 2153 -2162; d) J. Eichler, A. W. Lucka, R. A. Houghten, Peptide Res. 1994, 7, 300-307.

[11] R. Haubner, D. Finsinger, H. Kessler, Angew. Chem. 1997, 109, 1440 1456; Angew. Chem. Int. Ed. Engl. 1997, 36, 1374-1389; zit. Lit.

[12] S. R. Chhabra, B. Hothi, D. J. Evans, P. D. White, B. W. Bycroft, W. C. Chan, Tetrahedron Lett. 1998, 39, 1603-1606.
[13] H. Kunz, C. Unverzagt, Angew. Chem. 1984, 96, 426-427; Angew. Chem. Int. Ed. Engl. 1984, 23, 436-437.

[14] A. Schleyer, M. Meldal, M. Renil, H. Paulsen, K. Bock, Angew. Chem. 1997, 109, 2064-2067; Angew. Chem. Int. Ed. Engl. 1997, 36, $1976-$ 1978.

[15] a) U. Ellervik, G. Magnusson, Carbohydr. Res. 1996, 280, 251-260; b) M. Schultz, H. Kunz, Tetrahedron: Asymmetry 1993, 4, 1205-1220.

[16] P. Sieber, Tetrahedron Lett. 1987, 28, 2107-2110.

[17] D. A. Wellings, E. Atherton, Methods Enzymol. 1997, 289, 44-67.

[18] B. W. Bycroft, W. C. Chan, S. R. Chhabra, N. D. Hone, J. Chem. Soc. Chem. Commun. 1993, 778-779.

[19] S. C. Story, J. V. Aldrich, Int. J. Pept. Protein Res. 1994, 43, 292-296.

[20] Bei dieser Cyclisierungsmethode wurde der Rink-Amid-Linker zur Ankupplung an das Harz verwendet und die $\varepsilon-\mathrm{NH}_{2}$-Gruppe des am Ringschluss beteiligten Lysins mit der Mtt-Gruppe geschützt und nach Bildung des Pfp-Esters freigesetzt: D. Tumelty, M. C. Needels, V. V. Antonenko, P. R. Bovy in Peptides: Chemistry, Structure, and Biology (Hrsg.: P. T. P. Kaumaya, R. S. Hodges), Mayflower Scientific, Kingswinford, 1996, S. 121-122.

[21] Mit 2-proz. Hydrazinhydrat konnten selbst nach fünfmaliger Behandlung des Syntheseharzes noch deutliche Mengen des Abspaltprodukts 3-Isobutyl-6,6-dimethyl-4-oxo-4,5,6,7-tetrahydro- $1 H$-indazol UV-spektroskopisch $\left(A_{290}\right)$ nachgewiesen werden.

[22] E. Kaiser, R. L. Colescott, C. D. Bossinger, P. I. Cook, Anal. Biochem. 1970, 34, 595-598.

[23] Beobachtung einzelner Harzkugeln unter einem Mikroskop.

[24] Die Stabilität einer Glycosidbindung wird durch Acylierung des Kohlenhydrats erhöht: H. Kunz, C. Unverzagt, Angew. Chem. 1988, 100, 1763-1765; Angew. Chem. Int. Ed. Engl. 1988, 27, 1697.

[25] Verwendete Abkürzungen: Aloc $=$ Allyloxycarbonyl, $\mathrm{Bal}=\beta$-Alanin, Boc $=$ tert -Butoxycarbonyl, Dde $=1$-(4,4-Dimethyl-2,6-dioxocyclohexyliden)ethyl), $\quad \mathrm{Ddv}=1$-(4,4-Dimethyl-2,6-dioxocyclohexyliden)isovaleryl, DIEA = Ethyldiisopropylamin, ESI-MS = Elektrosprayionisations-Massenspektrometrie, Fmoc =9-Fluorenylmethoxycarbonyl, HBTU $=O$-Benzotriazol-1-yl- $N, N, N^{\prime}, N^{\prime}$-tetramethyluroniumhexafluorophosphat, HOBt $=1$-Hydroxybenzotriazol, Mtt $=$ (4-Methylphenyl)diphenylmethyl), NMP =1-Methyl-2-pyrrolidon, $\mathrm{MeOTf}=$ Trifluormethansulfonsäuremethylester, Pfp = Pentafluorphenyl, RPHPLC $=$ Umkehrphasen-HPLC,$\quad$ TBDPS $=$ tert -Butyldiphenylsilyl, TFA $=$ Trifluoressigsäure, Troc $=2,2,2$-Trichlorethoxycarbonyl. 